\title{
The start of the Abiogenesis: Preservation of homochirality in proteins as a necessary and sufficient condition for the establishment of the metabolism
}

\author{
Søren Toxvaerd \\ Department of Science and Environment, Roskilde University, Postbox 260, DK-4000 \\ Roskilde, Denmark
}

\begin{abstract}
Biosystems contain an almost infinite amount of vital important details, which together ensure their life. There are, however, some common structures and reactions in the systems: the homochirality of carbohydrates and proteins, the metabolism and the genetics. The Abiogenesis, or the origin of life, is probably not a result of a series of single events, but rather the result of a gradual process with increasing complexity of molecules and chemical reactions, and the prebiotic synthesis of molecules might not have left a trace of the establishment of structures and reactions at the beginning of the evolution. But alternatively, one might be able to determine some order in the formation of the chemical denominators in the Abiogenesis. Here we review experimental results and present a model of the start of the Abionenesis, where the spontaneous formation of homochirality in proteins is the precondition for the establishment of homochirality of carbohydrates and for the metabolism at the start of the Abiogenesis.
\end{abstract}

Keywords:

Abiogenesis Homochirality Metabolism

\section{The preservation of homochiral structures in millions of years in a prebiotic aqueous environment.}

There have been many proposals to the establishment of homochirality of carbohydrates and amino acids in a prebiotic environment. It is, however, not the establishment, but rather the preservation of homochirality, which is the problem. Both the units of amino acids in the proteins, as well as the central 
molecules, Glyceraldehyde and Glyceraldehyde-3-phosphate, in the polymerization of carbohydrates have active isomerization kinetics [1, 2], and this will, without other chemical constraints, give racemic compositions in aqueous solutions [3]. Biosystems are from a physicochemical point of view, soft condensed matter in aqueous environments, and the Abiogenesis must have taken millions of years, so the establishment of homochirality should in fact be readdressed to: the establishment and preservation of homochiral structures in millions of years in an aqueous environment.

The chiral composition and stability of a system of chiral units is determined by the ratio between a decrease in entropy, and thereby an increase of Gibbs free energy by homochiral ordering, $(\approx 2 \mathrm{~kJ} / \mathrm{mol})$, and the gain in enthalpy by a chiral discrimination in favour of homochirality [4, 5, 3]. The chiral discrimination is given by the difference in enthalpy, $\Delta H_{\mathrm{CD}}$, between the enthalpy, $\Delta H_{\mathrm{f}}$ of a homochiral system, $(L)$, and a corresponding racemic mixture, $(D L)$. The chiral discrimination must be negative and and less than $\div 2 \mathrm{~kJ} / \mathrm{mol}$ in order overcome the loss of entropy and to establish homochiral domains. The formation enthalpy, for for crystals, (c), $\Delta H_{\mathrm{f}}^{\ominus}(c)$, can be determine from combustion energy, and the chiral discrimination is

$$
\Delta H_{\mathrm{CD}}^{\ominus}(c)=\Delta H_{\mathrm{f}}^{\ominus}(c, L)-\Delta H_{\mathrm{f}}^{\ominus}(c, D L) .
$$

A chiral system in an aqueous environment and with isomerization kinetics and chiral discrimination of its chiral units tends to a homochiral ordering at high concentrations of its chiral units and low water activity, but racemizes at higher water activity. The solution in a cell (cytosol) can be characterized as an aqueous-like solution with an emulsion of proteins, and in a recent article [3] it was argued, that the establishment of homochirality was started and preserved in the hydrophobic core of enzyme-like proteins. The conclusion was obtained from thermodynamic considerations and from Molecular Dynamics simulations of a model of peptide chains in aqueous solutions. Here we shall review and summarize experimental results which support this hypothesis. The spontaneous formation of homochiral proteins was, however, also a sufficient, but necessary condition for the establishment of homochirality of the carbohydrates, and the metabolism at the start of the Abiogenesis. 


\section{The establishment and preservation of homochiral structures in proteins.}

The structures of proteins were predicted by Linus Pauling in a series of papers in 1951, where he derived the two secondary structures of proteins, the $\alpha$-helix [6] and the pleated $\beta$-sheet conformation [7]. The clockwise (positive) $\alpha$-helix structure was derived from spectroscopic data for bond lengths and angles at the planar peptide units, and with an intramolecular stabilizing hydrogen-bond between a hydrogen atom at the substituted -N$\mathrm{H}$ and an oxygen atom in a $-\mathrm{C}=\mathrm{O}$ group in the helix. The energy of the $-\mathrm{N}-\mathrm{H} \cdots \mathrm{O}=\mathrm{C}$ - hydrogen bond $(\approx 10 \mathrm{~kJ} / \mathrm{mol})$ is only of the order a half of the energy of a corresponding intermolecular hydrogen bond [8], and this fact has important consequences. The loss of intermolecular hydrogen bond energy by the establishment of the peptide bonds results in an emulsion-like aqueous phase separation with compact globular proteins, where the weaker intramolecular hydrogen bonds stabilize the secondary protein structures.

The proposed $\alpha$-helix (Figure 2 in [6] ) is, however, not the clockwise all L- $\alpha$-helix structure, but its mirror structure, an anticlockwise all D-helix. [9] . This mix of notation was irrelevant for Linus Pauling and for the contents of his article, but might be relevant for understanding the ability to maintain homochirality in the hydrophobic compact part of the globular protein. The two helices have the same minimum intramolecular energy (enthalpy), and although one cannot conclude, that helix(+)- structures with a more racemic composition and with even lower energy does not exist, it is not likely. The enthalpy of a protein is given by the intramolecular energies, including the energy from the intramolecular hydrogen bonds and the "hydrophobic forces" (surface tension) from the loss of intermolecular hydrogen bonds by the phase separation, which all together ensure the compact core and a sufficient chiral discrimination for the homochirality. However, some bioproteins with a few units of D-amino acids exist [10], which indicates that homochirality in proteins, although it stabilizes the compact helical state [11], is not a strict necessity for the establishment of the secondary structures in proteins.

The active isomerization kinetics in proteins affects the stability of the structure, and the aging of proteins in the cells results in appearance of units

of D-amino acids, and with the loss of higher order structures [12]. The homochirality in proteins is in [3] predicted to be more stable at a smaller water activity, e g. at higher ionic concentrations than in the cytosol solution in biological cells. 
TABLE I: Chiral discrimination, $\Delta H_{\mathrm{CD}}^{\ominus}(c)$

$\begin{array}{lcl}\text { Molecule } & \Delta_{\mathrm{CD}} H^{\ominus}(c) \mathrm{kJ} / \mathrm{mol} & \text { References } \\ \text { Alanine } & 4.3 & {[13,[14,15]} \\ \text { Valine } & 2.4 & {[16]} \\ \text { Leucine } & 3.4 & {[17} \\ \text { Isoleucine } & -5.4 & {[18,19]} \\ \text { Serine } & 7.4 & {[20]} \\ \text { Threonine } & -12.0 & 21 \\ \text { Proline } & 3.4 & {[22]} \\ \text { Tryptophan } & 1.3 & {[23,24]}\end{array}$

An indirect support of the hypothesis, that it is the secondary and tertiary homochiral structures of a protein which ensure the homochirality, can be obtained from the strength of the chiral discrimination of the individual amino acids. An updated table with recent data for the chiral discrimination of crystals of amino acids is given in Table I. The chiral discriminations, $\Delta H_{\mathrm{CD}}^{\ominus}(c)$, are obtained from Eq. 1 and data for $\Delta H_{\mathrm{f}}^{\ominus}(c, L)$ and $\Delta H_{\mathrm{f}}^{\ominus}(c, D L)$. Only data for less than half of the twenty biological $\alpha$-amino acids exists. But one can, however, see from the table that most amino acids have a positive chiral discrimination, i.e. in favor of a racemic crystallization of the amino acids. Only two (Isoleucine and Threonine) out of the eight amino acids have a sufficient negative chiral discrimination to favour homochirality in solid state. In an aqueous solution the chiral discrimination is, however, less (negative) [3].

The homochiral stability of proteins cannot be explained by a direct chiral discriminations between units of amino acids in aqueous solutions, but must originate from the $\alpha$-amino acids ability to perform proteins with higher order compact and stable structures, when they are homochiral. This property can also explain, why there is no direct relation between the sequence of the units of L-amino acids in an enzyme, the secondary structure and its biological function. The sequence of the units in a given enzyme differs in biosystems, and for homologous enzymes these differences can be used to obtain the cladistics of the biological evolution [25, 26]. The sequence in 
e.g. insulin is on one hand specific for a given biological system [27], but its tertiary conformation, and thereby the enzymatic function in the metabolism is on the other hand maintained to a degree, that an insulin molecule in one biosystem can function in another biosystem.

\section{The establishment and preservation of homochiral structures in carbohydrates.}

Theformation of a compact hydrophobic core in a protein can explain the stability of homochiral proteins [3], but one still has to explain the formation and stability of the D- polysaccharides in a prebiotic aqueous environment. The central chiral molecule in the formose reaction for spontaneous synthesis of carbohydrates [28] is Glyceraldehyde, and the corresponding key molecule in the Glycolysis is Glyceraldehyde-3-phosphate. Both molecules have an active isomerization kinetics in an aqueous solution,

$$
\begin{aligned}
& \text { D-Glyceraldehyde-3-phosphate } \rightleftharpoons \text { Dihydroxyacetone phosphate } \\
& \text { Dihydroxyacetone phosphate } \rightleftharpoons \text { L-Glyceraldehyde-3-phosphate }
\end{aligned}
$$

and this will, without other chemical constraints, give a racemic composition at relevant biological concentrations [3]. Furthermore all living systems contain a very effective enzyme, Triose Phosphate Isomerase, which eliminates the activation energy in the isomerization kinetics of Glyceraldehyde3-phosphate [29]. This simple enzyme is believed to have been present in LUCA (Last Universal Common Ancestor, also called the Last Common Unicellular Ancestor) and could be among the very first enzymes in the evolution [30, 31]. The enzyme will, without further physicochemical constraints reinforce the formation of a racemic composition of carbohydrates in biosystems. So the presence of this enzyme indicates, that the homochirality of the carbohydrates in biosystems is not obtained by discrimination, but is established and preserved by another physicochemical mechanism.

To understand the formation and preservation of homochirality in polysaccharides and in the metabolism, one must notice, that these chain reactions are reversible. Take for instant the central step in the Glycolysis,

$$
\begin{array}{r}
\text { D-Fructose-1,6-diphosphate } \rightleftharpoons \\
\text { Dihydroxyacetone phosphate } \\
+ \text { D-Glyceraldehyde-3-phosphate. }
\end{array}
$$




\section{Enzyme DD}
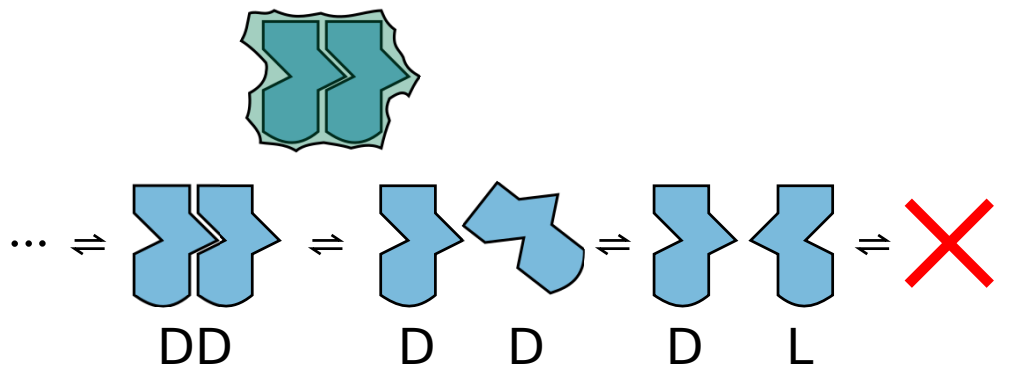

Figure 1: Illustration of a chain reaction with a stereospecific enzymatic (Enzyme $\mathrm{E}_{\mathrm{DD}}$ ) catalyse of one of the steps in the chain reaction. The figure illustrates polymerization of a homochiral polymer from chiral monomers with isomerization kinetics and a stereospecific catalyse of the first step of the polymerization into a dimer DD. In the illustration there is no enzymes, Enzyme $\mathrm{E}_{\mathrm{DL}}$ and Enzyme $\mathrm{E}_{\mathrm{DD}}$, and therefore no polymerization into a non-homochiral polymer.

Although $\Delta G^{\ominus^{\prime}}=23.97 \mathrm{~kJ} / \mathrm{mol}$ of this reaction is strongly positive, it readily proceeds in the forward direction under intracellular conditions $(\approx$ $10-100 \mu \mathrm{M})$, and with a Gibbs free energy gain, $\Delta_{r} G<0$, by cleaving Fructose. But the reverse reaction is spontaneous at higher of concentrations of Dihydroxyacetone phosphate and D-Glyceraldehyde 3-phosphate, and with a gain of free energy by synthesis of Fructose and a storage of free energy in Frucose-6-phosphate, and in the first component, Glycose-6-phosphate, in the Glycolysis.

The isomerization kinetics at the chiral carbon atom in Glyceraldehyde is, however, not active after the condensation of Glyceraldehyde and Dihydroxyacetone, and the original chirality is conserved by the condensation. The pentoses and hexoses contain additional chiral carbon atoms, created by this and other synthesis, and with a keto-enol isomerization between them. But the chirality at carbon atom No. 5 (No. 4 position for pentoses) is not affected by these keto-enol isomerizations. The homochiral structures are not limited to simple D-polysaccharides, also the chains in RNA and DNA are all D-structures of phosphate esters of Ribose and Deoxyribose, respectively. D-Ribose-5-phosphate has a metabolism which is linked to the Glycolyse, and altogether these facts can explain the formation and preservation of a homochiral carbohydrate world: $d$.

If there at the establishment of the consecutive reactions in the metabolism 
was an enzyme, which only reduced the activation energy for the reaction with the D-structure of the carbohydrate, but not for the L-structure, then a metabolism will drain the aqueous solution with the central units, Glyceraldehyde-3-phosphate, for the D-conformations. The active isomerization kinetic will convert L-Glyceraldehyde-3-phosphate to D-Glyceraldehyde3 -phosphate and ensure a racemic equilibrium of the two enantiomers in the aqueous solution. But the net result will be a dominance of D-carbohydrates. Such enzymes do in fact exist. The enzyme Hexokinase (Glucokinase), which catalyses the first step in the Glycolysis, only catalyses phosphorylation of DGlycose, but not L-Glycose. The enzyme Ribokinase, which phosphorylates D-Ribose does not catalyse the corresponding phosphorylation of L-Ribose [32]. The first step in the Glycolysis is the stereospecific phosphorylation of D-Glycose to D-Glycose-6-phosphate. RNA and DNA, are correspondingly phosphor polyesters, so the phosphorylation is central for the establishment of the metabolism as well as the genetics. An explanation of the role of the phosphorylation in the Abiogenesis is given by Westheimer [33], who argued that the phosphorylation stabilizes the biomolecules. A phosphorylation must have appeared at the establishment of a metabolism. If a stereospecific proteins like Hexokinase and Ribokinase by changes were present at the prebiotic phosphorylation of the carbohydrates, it is sufficient to ensure the homochirality of carbohydrates in the metabolism.

An illustration of reactions with chiral objects, and with isomerization kinetics between the chiral units and a stereoselectiv enzymatic catalyse of a step in the chain of reactions, is shown in Figure 1. The figure illustrates a polymerization of chiral units. A homochiral protein with a secondary structure has a stereospecific surface, which can catalyse a chemical reaction. In the illustration there is only a surface which catalyses the $\mathrm{D}+\mathrm{D}$ dimerisation, whereas there is no stereospecific surfaces for $\mathrm{D}+\mathrm{L}$ or $\mathrm{L}+\mathrm{L}$ dimerizations. If a prebiotic environment is enriched with the monomers, in the case of carbohydrates with Glyceraldehyde by the formose reaction, and polymerizes, then the isomerization kinetics between the chiral units together with a D-stereospecific enzyme somewhere in the chain reactions will ensure a dominating D-world.

A prebiotic existence of stereospecific proteins for a reaction in the metabolism can explain the formation and stability of the homochiral carbohydrate world. But it also establish an order in the evolution at the start of the Abiogenesis. The creation of a prebiotic peptide world with, by change, a stereospecific enzymatic protein for a step in the metabolism is sufficient for the establish- 


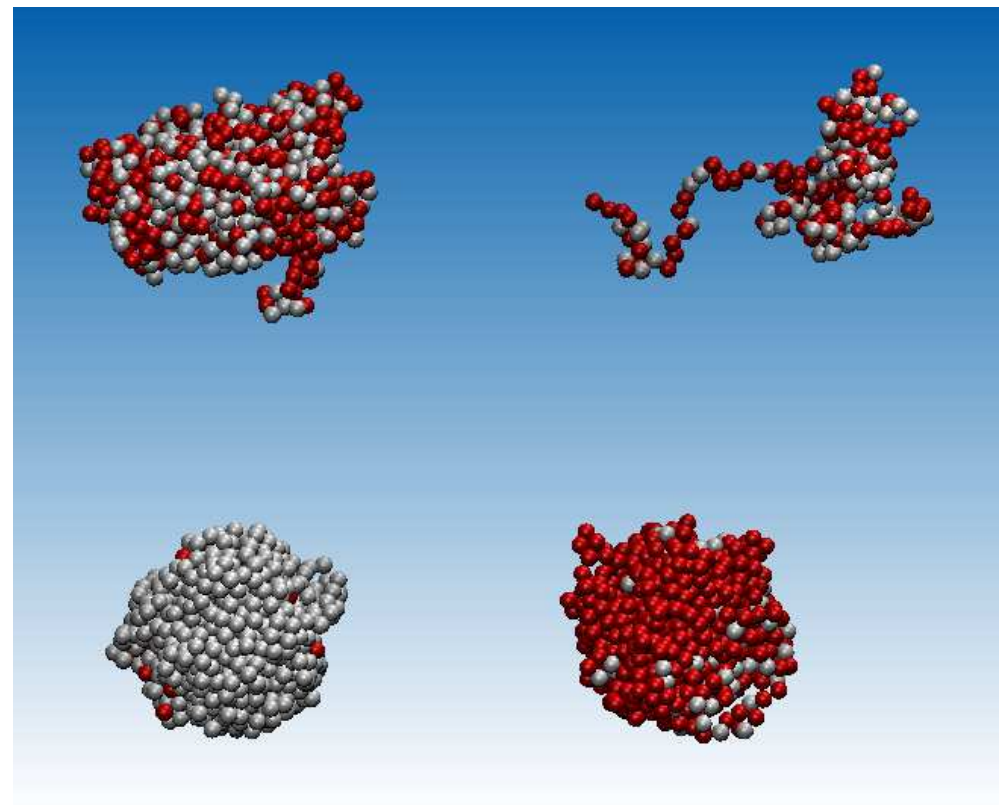

Figure 2: Illustration of an aqueous emulsion of proteins. The four proteins are from the simulations of peptide chains with isomerization kinetics and in aqueous solutions [3]. The activity of water in the solution is given by the blue colour (blue: high activity; white: low activity). The units of amino acids can have different chirality: red (L) and white (D). The peptide chain (upper right) consists of 200 units and remained with a racemic composition during the simulation. The peptides with longer chains, (1000 units), had a racemic distribution of the chiral units for a high water activity (upper left), but for lower activity the isomerization kinetics resulted in compact proteins with homochiral compositions, but with a statistical equal amount of proteins with either D- (lower left)or L- chirality (lower right).

ment of a homochiral carbohydrate world.

\section{The establishment and preservation of the L-protein and D- carbohydrate world.}

Linus Pauling's anticlockwise all D-peptide helices must be the key-molecules in a D-protein- and L-carbohydrate world, a world which not exists on planet Earth. In [4, 5, 3] it was pointed out, that one can obtain a spontaneous symmetry break in a racemic system and a dominance of one of the chiral units, when the subdomain of one of the homochiral conformations percolates the 
system. But the compact proteins in an aqueous emulsion are autonomous, and the isomerization kinetics can one hand ensure homochirality in the proteins, but will on the other hand give an statistical equal amount of homochiral proteins with either D- or L-units. Enzymes are compact proteins with from 62 to $\approx 2500$ amino acids. Computer simulations of simple peptide chains in an aqueous environment and with isomerization kinetics show, that short chains remained in an open structure with a racemic composition, whereas longer chains had a compact structure and they were almost homochiral for low water activity [3]. Figure 2 show four proteins: a relative short peptide chain with 200 units (upper right) and four proteins with 1000 units. A prebiotic aqueous emulsion of proteins and without a selective mechanism will contain both homochiral D-and L-proteins and not be global homochiral.

There is, however, a crucial quality of the metabolism and catabolism of carbohydrates and peptides, which can explain the emergence and preservation of the L-protein and D-carbohydrate world only. The L-carbohydrates are not included in the metabolism of carbohydrates, but simple bacteria [34] and procariotes [35] contain enzymes, which catalyse a catabolic pathway for L-glycose. The metabolism of amino acids contains enzymes, which cleave the peptide bonds, and which are not stereosellective, e.g. Trypsin and Pepsin, and enzymes, which include D-amino acids in the catabolism, e.g. D-amino acid dehydrogenase [36]. The critical barrier for growth and stability of one of the chiral worlds must be the establishment of its metabolism and catabolism. Who came first will eat the other by the catabolism.

\section{The Abiogenesis.}

The Abiogenesis, or the origin of life, is probably not a result of a series of single events, but rather the result of a gradual process with increasing complexity of molecules and chemical reactions. This article argues, however, that there are some "milestones" and order in the evolution of the complex molecular biostructures and networks of chemical reactions.

One of the elements in the Abiogenesis is the establishment by time of an aqueous emulsion of homochiral compact proteins. The homochirality can be obtained by the isomerization kinetics and preserved by the compact secondary and tertiary structures of the proteins in aqueous emulsions with low water activity (e.g. high ionic concentrations) [3]. 
Another link in the evolution is the establishment of a metabolism. The first step in the Glycolysis is the stereospecific phosphorylation of Glucose to D-Glucose-6-phosphate. RNA and DNA, are correspondingly phosphor polyesters, so the phosphorylation is central for the establishment of the metabolism as well as the genetics. The synthesis of peptides and carbohydrates have appeared simultaneously in the prebiotic aqueous environment. A stereospecific phosphorylation can be obtained by proteins, which by change acted as enzymes for phosphorylation of D-glucose and D-Ribose only and thereby established the D-carbohydrate world. Whereas one today can find a few examples of D-amino acid units in biosystems, L-carbohydrates does not appear. The explanation for this lack of L-carbohydrates and dominance of peptides with units of $\mathrm{L}$ amino acids today can very well be the same as the explanation for the formation and preservation of homochiral carbohydrates given here: the existence of the stereospecific enzymes like Hexokinase and Ribokinase together with a stereospecific catabolism of Lcarbohydrates is sufficient to establish the homochirality in the metabolism and to remove the L-carbohydrates and proteins with D-units.

Can this order in the evolution be verified? Perhaps not, the prebiotic world has disappeared on planet Earth. But there is a chance that frozen ice in cavities in the rocks beneath the evaporated oceans on planet Mars contains prebiotic materials, which can reveal the start of the Abiogenesis.

\section{Acknowledgment}

Jeppe C Dyre and Ulf R Pedersen are gratefully acknowledged. This work was supported by the VILLUM Foundations Matter project, grant No. 16515

\section{References}

[1] Bada J.L., 1972. Kinetics of razimization of amino acids as a function of pH. J. Am. Chem. Soc. 94, 1371-1373.

[2] Nagorski R.W., Richard J.P., 2001. Mechanistic imperatives for aldoseketose isomerization in water: specific, general base- and metal ioncatalyzed isomerization of glyceraldehyde with proton and hydride transfer. J. Am. Chem. Soc. 123, 794-802. 
[3] Toxvaerd, S., 2017. The role of the peptides at the origin of life. J. Theor. Biol. 429, 164-169.

[4] Toxvaerd, S., 2000. Molecular dynamics simulations of isomerization kinetics in condensed fluids. Phys. Rev. Lett. 85, 4747-4750 .

[5] Toxvaerd, S., 2009. Origin of homochirality in biosystems. Int. J. Mol. Sci. 10, 1290-1299 .

[6] Pauling, L., Corey R.B., Branson, H.R., 1951. The structure of proteins: Two hydrogen-bonded helical configurations of the polypeptide chain. Proc. Natl. Acad. Sci. 37, 205-211.

[7] Pauling, L., Corey R.B., 1951. The pleated sheet, a new layer configuration of polypeptide chains. Proc. Natl. Acad. Sci, 37, 251-256.

[8] Wendler, K., Thar, J., Zahn, S., Kirchner, B., 2010. Estimating the hydrogen bond energy. J. Phys. Chem. A 144, 9529-9536.

[9] Dunitz, J.D., 2001. Pauling's left-handed $\alpha$-helix. Angew. Chem. Int. Ed. 40, 4167-4173.

[10] Fujii, N., 2002. D-amino acids in living higher organisms. Origins Life Evold. Biosphere 32, 103-127.

[11] Nanda, V., Andrianarijaona, A., Narayanan, C., 2007. The role of protein homochirality in shaping the energy landscape of folding. Protein Science 16, 1667-1675.

[12] Fujii, N., Fujii, N., Kida, M., Kinouchi, T., 2010. Influence of L $\beta$-, D $\alpha-$ and $\mathrm{D} \beta$-Asp isomers of the Asp-76 residue on the properties of $\alpha \mathrm{A}$ crystallin 70-88 peptide. Amino Acids 39, 1393-1399.

[13] Contineanu, I.; Marchidan, D.I., 1984. The enthalpies of combustion and formation of D-alanine, L-alanine, DL-alanine, and $\beta$-alanine. Rev. Roum. Chim. 29, 43-48.

[14] Kamaguchi, A., Sato, T., Sakiyama, M., Seki, S., 1975. Enthalpies of combusion of organic compounds. III.* D-, L- and DL-alanines. Bull. Chem. Soc. Jpn. 48, 3749-3750. 
[15] Ribeiro da Silva, M.A.V., Ribeiro da Silva, M.D.M.C., Santos, A.F.L.O.M., Roux, M.V., Foces-Foces, C., Notario, R., Guzmán-Mejía, R., Juaristi, E., 2010. Experimental and computational thermodynamical study of $\alpha$-alanine (DL) and $\beta$-alanine. J.Phys. Chem. B 114, 1647116480 .

[16] Ribeiro da Silva, M.A.V., Ribeiro da Silva, M.D.M.C., Santos, L.M.N.B.F., 2000. Standard molar enthalpies of formation of crystalline L-, D- and DL-valine. J. Chem. Therm. 32, 1037-1043.

[17] )Huffman, H.M., Fox, S.W., Ellis, E.L., 1937. Thermal data. VII. The heats of combustion of seven amino acids. J. Am. Chem. Soc. 59, 21442150 .

[18] Wu, D., Zhu, Y., Gao, Z., Qu, S., 1993. Determination of combustion heat of some amino acids. Wuhan Daxue Xuebao Ziran Kexueban 78-82.

[19] Vasiliév, V.P., Borodin, V.A., Kopnyshev, S.B., 1991. Calculation of the standard enthalpies of combustion and of formation of crystalline organic acids and complexones from the energy contributions of atomic groups. Russ. J. Phys. Chem. (Engl. Transl.) 65, 29-32.

[20] Neacşu, A., Gheorghe, D., Contineanu, I., Tănăsescu, S., 2014. A thermochemical study of serine stereoisomers. Therm. Acta 595, 1-5.

[21] Contineanu, I., Neacşu, A., Gheorghe, D.,Tănăsescu, S., Perişanu Ş., 2013. The thermochemistry of threonine stereoisomers. Therm. Acta 563, 1-5 (2013).

[22] Filipa, A., Santos, L.O.M., Notario, R., Ribeiro da Silva, M.A.V., 2014. Thermodynamic and conformational study of proline stereoisomers. J. Phys. Chem. B 118, 10130-10141.

[23] Lukyanova, V.A., Druzhinina, A.I., Pimenova, S.M., Ioutsi, V.A., Buyanovskaya, A.G., Takazova, R.U., Sagadeyev, E.V., Gimadeev, A.A., 2017. Thermodynamic properties of L-tryptophan. J. Chem. Therm. 105, 44-49.

[24] Kochergina, L.A., Krutova, O.N., Damrina, K.V., 2014. Standard enthalpies of formation for L-tyrosine, DL-norleucine, DL-tryptophan, DL$\alpha$-alanyl-DL-norleucine, and products of their dissociation in aqueous solution. Russian J. Phys. Chem. A 89, 755-758. 
[25] Margoliash, E., 1963. Primary structure and evolution of cytochrome C. Proc. Nath. Acad. Sci. 50, 672-679.

[26] van Thoai, N., Roche, J., 1968. Homologous Enzymes and Biological Evolution. New York:Gordon \& Breach.

[27] Stretton, A.O.W., 2002. The first sequence: Fred Sanger and insulin. Genetics 162, 527-532.

[28] Butlerow, A., 1861. Formation synthetique dùne substance sucree. Compt. Rend. Acad. Sci. 53, 145147.

[29] Alberty, W.J., Knowles, J.R., 1976. Free-energy profile for the reaction catalyzed by triosephosphate isomerase. Biochemistry 15, 5627-5631.

[30] Sobolevsky, Y., Guimarães, R.C., Trifonov, E.N., 2013. Towards functional repertoire of the earliest proteins. J. Biomol. Struct. Dyn. 31, 1293-1300.

[31] de Farias, S.T., Rêgo, T.G., Jośe, M.V., 2016. A proposal of the proteome before the last universal common ancestor (LUCA). Int. J. Astrobiol. 15, 27-31.

[32] Chuvikovsky, D.V., Esipov, R.S., Skoblov, Y.S., Chupova, L.A., Muravyova, T.I., Miroshnikov, A.I., Lapinjoki, S, Mikhailopulo, I.A., 2006. Ribokinase from E. coli: Expression, purification, and substrate specificity. Bioorg. Med. Chem. 14, 6327-6332.

[33] Westerner, F.H., 1987. Why nature chose phosphates. Science 235, $1173-$ 1178.

[34] Shimizu, T., Takaya, N., Nakamura, A., 2012. An L-glucose catabolic pathway in Paracoccus species 43P. J. Biol. Chem. 287, 40448-40456.

[35] Yoshida, H., Yoshihara, A., Teraoka, M., Terami, Y., Takata, G., Izumori, K., Kamitori, S., 2014. X-ray structure os a novel L-ribose isomerase acting on a non-natural sugar L-ribose as its ideal substarate. FEBS J. 281, 3150-3164. 
[36] Olsiewski, P.J., Kaczorowski, G.J., Walsh, C., 1980. Purification and properties od D-amino acid dehydrogenase, an inducible membranbound iron-sulfor flavoenzyme from Escherichia Coli B. J. Biol Chem. 255, 4487-4494. 